\title{
Neural-network-based Path Planning Optimization
}

\author{
Huamin Zhang ${ }^{1, a}$ hua Che ${ }^{2, b}$ Di Chen ${ }^{3, c}$ \\ 1Department of Aerial Ammunition Engineering \\ 2,3Department of Aerial Instrument and Electric Engineering
}

The First Aeronautical Institute of Air Force,23\# Hangkong Road Shihe discrict Xinyang city, henan,China

azyrll@126.comb44075491@qq.com ccode.cd@163.com

Keywords: Unmanned Aerial Vehicle(UAV), Path Planning Optimization, Neural-network

Abstract. In this paper some Unmanned Aerial Vehicles are equipped with a fixed camera to conduct surveillance operations, the route planning method are presented using neural network method. The simulation results are presented to demonstrate the performance and computational efficiency of the method. As neutral network approximation does not require analytic derivatives, speed could be further enhanced, it could be quite useful for real -time optimization control.

\section{General Path Planning Optimization}

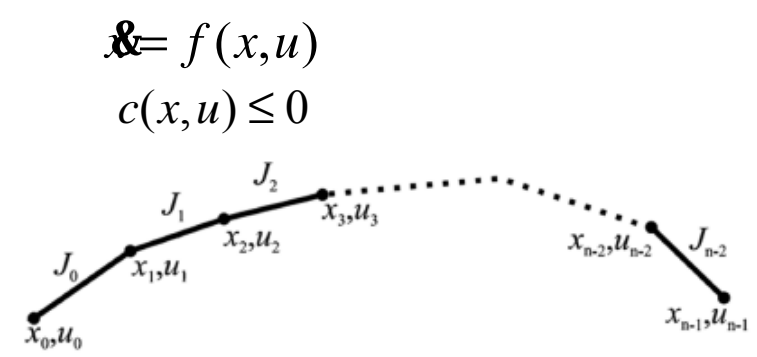

Fig1. sample trajectory

Where $\mathrm{x}$ and $\mathrm{u}$ are the state and control input vectors respectively. One seeks the control input $\mathrm{u}(\mathrm{t})$ that minimizes a scalar objective function $J$ of the form

$$
\int_{\text {to }}^{t_{f}} \gamma(x, u) d t
$$

The state at the end of the segment is found by integrating the equations of motion over the segment as shown in Eq.( 4)

$$
x\left(t_{0}+\tau\right)=\int_{t o}^{t_{0+\tau}} f(x(t), u(t)) d t
$$

\section{Neural-network Method Formulation}

The neural network approximation results in

$$
\begin{aligned}
& x_{1}=Y_{d}\left(x_{0}, u_{0}, u_{1}\right) \\
& x_{2}=Y_{d}\left(x_{1}, u_{1}, u_{2}\right)=Y_{d}\left[Y_{d}\left(x_{0}, u_{0}, u_{1}\right), u_{1}, u_{2}\right]
\end{aligned}
$$

The states at each node are recursively computed from $x_{0}$ and $u[0,1 \ldots n-1]$ :

$$
\begin{aligned}
x_{i+1}=Y_{d}\left(x_{i}, u_{i}, u_{i+1}\right) & \text { for } \mathbf{i} \in[0,1, \ldots . . n-2]
\end{aligned}
$$


Similarly, to approach the objective function, the neural network is trained to approximate the value of the objective along a segment. Again the value of the objective function along such a segment depends only on the initial state and the control history.

$$
\mathrm{J}_{0}=\int_{t o}^{t_{0+\tau}} \gamma(x, u) d t
$$

Thus the objective function value depends only on the initial state at the first node and the controls at each node:

$$
\mathrm{J}=\sum_{\mathrm{i}=0}^{\mathrm{n}-2} \mathrm{~J}_{\mathrm{i}}=\sum_{\mathrm{i}=0}^{\mathrm{n}-2} Y_{\mathrm{J}}\left(x_{\mathrm{i}}, u_{\mathrm{i}}, u_{\mathrm{i}+1}\right)
$$

\section{Derivative Calculation of Neutral Network}

The equation for network output $\mathrm{z}$ is

$$
\mathrm{z}=y_{0}\left\{W_{0} y_{h}\left[W_{0} y_{i}\left(W_{i} k+b_{i}\right)+b_{h}\right]+b_{0}\right\}
$$

Using the chain rule,the gradient of the entire network with respect to the inputs is easily computed.For a three-layer network, the gradient is

$$
\nabla \mathrm{z}=\mathrm{D}_{0} W_{0} \mathrm{D}_{h} W_{h} D_{i} W_{i}
$$

Where $\mathrm{D}_{[\mathrm{i}, \mathrm{h}, \mathrm{o}]}$ denote diagonal matrices

$$
\begin{aligned}
& \mathbf{J}_{\mathbf{i}}=Y_{\mathbf{J}}\left(\mathrm{x}_{\mathbf{i}}, \mathrm{u}_{\mathbf{i}}, \mathrm{u}_{\mathbf{i}+1}\right) \\
& \quad \text { for } \mathbf{i} \in[0,1, \ldots \ldots . \mathrm{n}-2]
\end{aligned}
$$

Where the functions $\mathrm{Yd}($.$) and \mathrm{YJ}($.$) are now approximated by neural networks. Equation (10) can$ be applied to calculate the gradient.

\section{UAV Path Planning optimization}

\section{Equations of Motion}

The state equations used in this path planning problem involve simple two-dimentional kinematics, as shown in Eq.(12). The states include north and east positions of the target(rxtgt and rytgt) and $\mathrm{UAV}$ (rx and ry ), the true airspeed $\mathrm{V}$, and the aircraft heading $\Psi$. The controls are longitudinal acceleration command ua and bank angle command $\mathbf{u}_{\Phi}$ :

$$
\begin{aligned}
& \hat{\mathrm{X}}_{\mathrm{X}}=\mathrm{V} \cos (\Psi)-\mathrm{V}_{\text {wind } \mathrm{N}} \quad \hat{\mathrm{y}}=\mathrm{V} \sin (\Psi)-\mathrm{V}_{\text {wind } \mathrm{E}} \\
& \notin=u_{\mathrm{a}} \quad \notin=\mathrm{g} \tan \left(\mathrm{u}_{\Phi}\right) / \mathrm{V}_{\mathrm{t}} \\
& \delta_{\mathrm{rgt}}=\mathrm{V}_{\mathrm{tgtN}} \quad \delta_{\mathrm{ggt}}=\mathrm{V}_{\mathrm{tgtE}}
\end{aligned}
$$

Constraints on the problem include stall airspeed and maximum airspeed ,bank angle limits ,and longitudinal acceleration limits:

$$
\begin{aligned}
& \mathrm{V}_{\text {min }}<\mathrm{V}<\mathrm{V}_{\text {max }} \quad \phi_{\text {min }}<\mathrm{u}_{\phi}<\phi_{\max } \\
& \mathrm{a}_{\text {min }}<\mathrm{u}_{\mathrm{a}}<\mathrm{a}_{\max }
\end{aligned}
$$

\section{Multiple Unmanned Aerial Vehicles, Fixed camera simulation}

(1) Stationary target

Figure 2 shows two UAVs observing a stationary target with no-fly zone. The question of collision avoidance is not addressed for this work. As a practical matter, flying the UAVs at different altitudes removes the need for collision avoidance. This would require the target-in-view network to be trained against altitude. For these results, all UAVs are flown at the same altitude. Note in Fig.3a how the black UAV diverts at the start to avoid simultaneous target coverage. The given coverage time is a $30 \mathrm{~s}$ moving average. Figure $2 \mathrm{~b}$ shows that the UAVs alternate sensor coverage as desired. The black and blue bars indicate when the sensor onboard UAV 1 or 2 is viewing the target. 


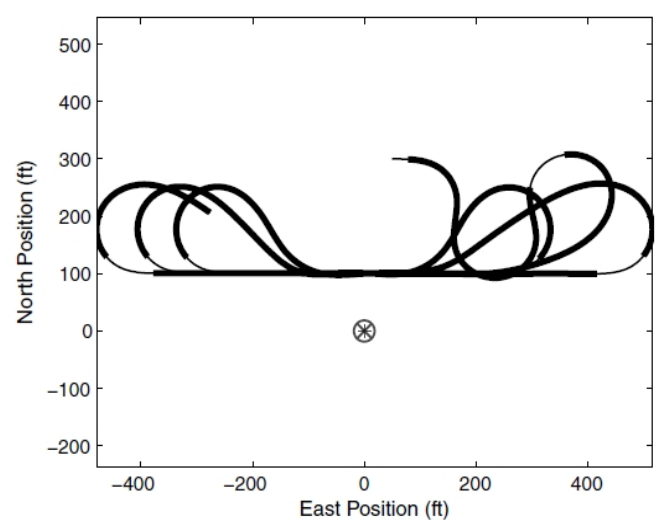

(a)Stationary target with no-fly zone (91.5\% coverage, 74 sec calculation time)

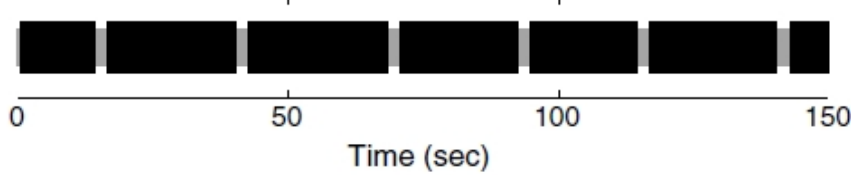

(b) stationary target with non-fly zone observation history

Fig.2 Stationary target with no-fly zone

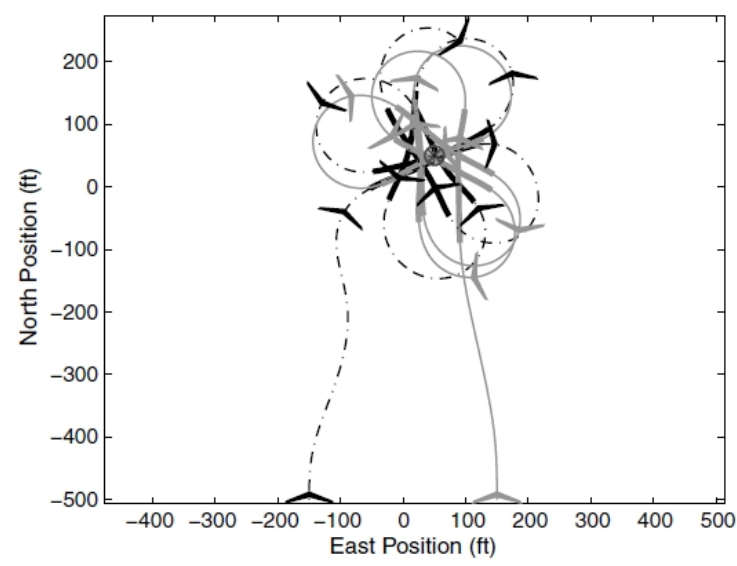

(a) Ground path( 9 nodes, $16 \mathrm{sec}$ horizon, $71.5 \%$ coverage, $0.60 \mathrm{sec}$ average calculation time)

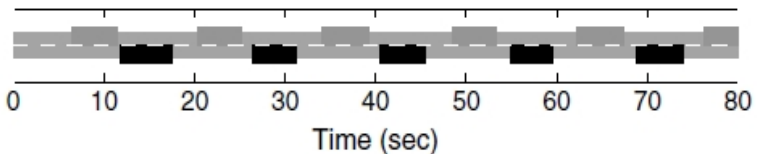

(b) Target coverage time line

Fig3. Ground track and coverage timeline for two UAVS and stationary target.

(2)Moving Target

A moving target is presented in Fig4.Here,the target is moving north at $15 \mathrm{ft} / \mathrm{s}$. After the initial settle time, the UAVs assume a regular pattern of alternating passes over the target. 


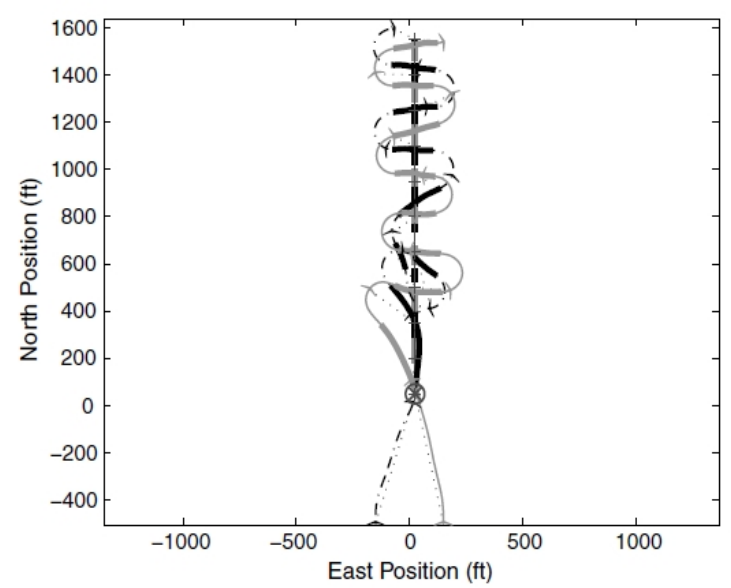

(a)Ground path( 9 nodes, $16 \mathrm{sec}$ horizon,91\% coverage, $0.58 \mathrm{sec}$ average calculation time)

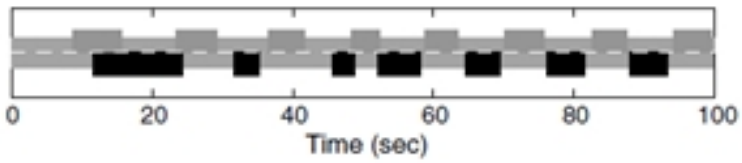

(b) Target coverage timeline

Fig. 4 Ground track and coverage timeline for two UAVs and a target at $15 \mathrm{ft} / \mathrm{s}$.

\section{Conclusion}

In this paper the neutral network path-planning optimization method is discussed. The results from the multiple UAV with a fixed camera have shown that neutral network approximation generally matches the optimization.

\section{References}

[1] Neutral Network-based Trajectory Optimization for Unmanned Aerial Vehicles ,Journal of Guidance Control and Dynamics,Vol.35,No.2,March-April 2012, Joseph F. Horn* and Eric M. Schmidt

[2] Geiger,B.R.,and Horn,J.F.,’Neural Network Based Trajectory Optimization for Unmanned Aerial Vehicles," 47th AIAA Aerospace Sciences Meeting, AIAA paper 2009-54,5-8 Jan.2009

[3] Geiger,B.R.schmidt,E.M. and Horn,'Use of Neural Network Approximation in Multiple-Unmanned Aerial Vehicle Trajectory Optimization", AIAA Guidance,Navigation and Control Conference, AIAA paper 2009-6003,Chicago,IL,10-13 Aug.2009

[4] Benson,D.A.,Huntington,G.T.,Thorvaldsen,T.p.and Rao,A.V.”Direct Trajectory Optimization and Costate Estimation via an orthogonal collocation Method",Journal of Guidance,Vontrol,and Synamics, Vol.29,No.6,2006,pp.1435-1440

[5] Fahroo,F.,abd Ross,M.I.,'Direct Trajectory Optimization by a Chebyshev Rseudospectral Method", Journal of Guidance,Vontrol,and Synamics,Vol.25,No.1,2002,pp.160-166 